\title{
INTERPOLATION OF THE ESSENTIAL SPECTRUM AND THE ESSENTIAL NORM
}

\author{
A. G. AKSOY \\ Department of Mathematics, Claremont McKenna College, Claremont, CA 91711, U.S.A. \\ E-mail: aaksoy@claremontmckenna.edu \\ H.-O. TYLLI \\ Department of Mathematics and Statistics, P.O. Box 68 (Gustaf Hällströmin katu 2b), \\ FIN-00014 University of Helsinki, Finland \\ E-mail: hojtylli@cc.helsinki.fi
}

\begin{abstract}
The behavior of the essential spectrum and the essential norm under (complex/real) interpolation is investigated. We extend an example of Albrecht and Müller for the spectrum by showing that in complex interpolation the essential spectrum $\sigma_{e}\left(S_{[\theta]}\right)$ of an interpolated operator is also in general a discontinuous map of the parameter $\theta$. We discuss the logarithmic convexity (up to a multiplicative constant) of the essential norm under real interpolation, and show that this holds provided certain compact approximation conditions are satisfied. Some evidence supporting a counterexample is presented.
\end{abstract}

Introduction. This note is concerned with the behaviour of the essential spectrum of an operator under complex interpolation and that of the essential norm under real interpolation. Let $\left(E_{0}, E_{1}\right),\left(F_{0}, F_{1}\right)$ be Banach interpolation couples and $T \in L\left(E_{0}+E_{1}\right.$, $\left.F_{0}+F_{1}\right)$ a compatible bounded linear operator, that is, the restrictions $T_{0}: E_{0} \rightarrow F_{0}$ and $T_{1}: E_{1} \rightarrow F_{1}$ are bounded operators. Let $\left(E_{0}, E_{1}\right)_{\theta, p}$ be the corresponding real interpolation space, where $0<\theta<1$ and $1 \leq p \leq \infty$. Then the restriction of $T$ defines a bounded operator $T_{\theta, p}:\left(E_{0}, E_{1}\right)_{\theta, p} \rightarrow\left(F_{0}, F_{1}\right)_{\theta, p}$ that satisfies the logarithmically convex bound

$$
\left\|T_{\theta, p}\right\| \leq\left\|T_{0}\right\|^{1-\theta}\left\|T_{1}\right\|^{\theta} .
$$

Estimate (1) also holds for $T_{[\theta]}:\left(E_{0}, E_{1}\right)_{[\theta]} \rightarrow\left(F_{0}, F_{1}\right)_{[\theta]}$ between the corresponding complex interpolation spaces for $0<\theta<1$. We refer to e.g. [BL], [BS] or [KM] for the definitions and the basic properties of real and complex interpolation.

2000 Mathematics Subject Classification: 46B70, 47A10, 47A30.

Research of H.-O. Tylli supported by the Academy of Finland Project \# 53893.

The paper is in final form and no version of it will be published elsewhere. 
Let $K(E, F)$ denote the compact operators $E \rightarrow F$, where $E, F$ are Banach spaces. The essential norm

$$
\|S\|_{e}=\operatorname{dist}(S, K(E, F)), \quad S \in L(E, F),
$$

is the quotient norm in $L(E, F) / K(E, F)$. If $E$ is a complex Banach space, then the essential spectrum of $S \in L(E)$ is

$$
\sigma_{e}(S)=\sigma(S+K(E)),
$$

the spectrum of $S+K(E)$ in the Calkin algebra $L(E) / K(E)$.

Recently Albrecht and Müller $[\mathrm{AM}]$ gave an example of a couple $\left(X_{0}, X_{1}\right)$ and a compatible operator $T \in L\left(X_{0}+X_{1}\right)$ for which the map $\theta \mapsto \sigma\left(T_{[\theta]}\right)$ is discontinuous at an interior point $\theta \in(0,1)$ in complex interpolation. It is important to have an analogous example for the essential spectrum, which is a useful subset of the spectrum of a bounded operator that is related to Fredholm theory. It turns out (Proposition 1 below) that the example of Albrecht and Müller already exhibits a similar discontinuity for the essential spectrum.

We also study the logarithmic convexity (up to a multiplicative constant) of $\left\|T_{\theta, p}\right\|_{e}$ under real interpolation, and establish positive results under certain compact approximation conditions. One purpose of this note is to draw attention to the intriguing open problem whether $\left\|T_{\theta, p}\right\|_{e}$ is logarithmically convex in general. We discuss some facts supporting a counterexample, and include some examples of the surprising behaviour of $\|\cdot\|_{e}$ under isometric embeddings and metric surjections.

Discontinuity of the essential spectrum in complex interpolation. We start by recalling the required details from [AM, pp. 808-809]. The spaces $\left(X_{j},\|\cdot\|_{j}\right)$ for $j=0,1$ are the (weighted) Hilbert spaces consisting of the scalar sequences $x=\left(a_{j}\right)_{j \in \mathbf{Z}}$ for which

$$
\left\|\left(a_{j}\right)\right\|_{0}=\left(\sum_{j \in \mathbf{Z}} 2^{-2 j}\left|a_{j}\right|^{2}\right)^{1 / 2}<\infty \quad \text { and } \quad\left\|\left(a_{j}\right)\right\|_{1}=\left(\sum_{j \in \mathbf{Z}} 2^{2 j}\left|a_{j}\right|^{2}\right)^{1 / 2}<\infty .
$$

Let $\left(e_{n}\right)_{n \in \mathbf{Z}}$ be the unit coordinate basis, so that $\left\|e_{n}\right\|_{0}=2^{-n}$ and $\left\|e_{n}\right\|_{1}=2^{n}$ for $n \in \mathbf{Z}$. The complex interpolation spaces $\left(X_{0}, X_{1}\right)_{[\theta]}$ are the (weighted) Hilbert spaces consisting of the scalar sequences $x=\left(a_{j}\right)=\sum_{j \in \mathbf{Z}} a_{j} e_{j}$, for which

$$
\left\|\sum_{j \in \mathbf{Z}} a_{j} e_{j}\right\|_{[\theta]}=\left(\sum_{j \in \mathbf{Z}} r_{\theta}^{2 j}\left|a_{j}\right|^{2}\right)^{1 / 2}<\infty
$$

where $r_{\theta}=2^{-(1-\theta)} 2^{\theta}=2^{2 \theta-1}$. Note that $r_{1 / 2}=1$, so that $\left(X_{0}, X_{1}\right)_{[1 / 2]}=\ell^{2}(\mathbf{Z})$. Let $H_{0}=\ell^{2}\left(\mathbf{Z}, X_{0}\right)$ and $H_{1}=\ell^{2}\left(\mathbf{Z}, X_{1}\right)$ be the vector-valued direct $\ell^{2}$-sums indexed by $\mathbf{Z}$. It follows that $\left(H_{0}, H_{1}\right)_{[\theta]}=\ell^{2}\left(\mathbf{Z},\left(X_{0}, X_{1}\right)_{[\theta]}\right)$ for $0<\theta<1$.

Let $S: X_{j} \rightarrow X_{j}$ be the (weighted) right shift operator $S e_{k}=e_{k+1}$ for $k \in \mathbf{Z}$ and $j=0,1$. Note that $\|S x\|_{0}=\frac{1}{2}\|x\|_{0}$ for $x \in X_{0}$ and $\|S x\|_{1}=2\|x\|_{1}$ for $x \in X_{1}$. Thus $S_{[\theta]}:\left(X_{0}, X_{1}\right)_{[\theta]} \rightarrow\left(X_{0}, X_{1}\right)_{[\theta]}$ is defined by the same condition, and $\left\|S_{[\theta]} x\right\|_{[\theta]}=r_{\theta}\|x\|_{[\theta]}$ for $x \in\left(X_{0}, X_{1}\right)_{[\theta]}$ and $0<\theta<1$. Define $T \in L\left(H_{0}+H_{1}\right)$ by

$$
T\left(x_{j}\right)=\left(\ldots, S x_{-2}, S x_{-1},(S-I) x_{0}, S x_{1}, \ldots\right),
$$


where $S x_{-1}$ is the 0 -th coordinate. It was verified in [AM, Example 1] that $\sigma\left(T_{[\theta]}\right)=$ $\left\{\lambda \in \mathbf{C}:|\lambda|=r_{\theta}\right\}$ for $0<\theta<1$ and $\theta \neq \frac{1}{2}$, and that $\sigma\left(T_{[1 / 2]}\right)=\{\lambda \in \mathbf{C}:|\lambda| \leq 1\}$. The aim of this section is to show that there is a similar discontinuity for the essential spectrum.

Let $E, F$ be Banach spaces. Recall that $S \in L(E, F)$ is a Fredholm operator, denoted by $S \in \Phi(E, F)$, if its kernel $\operatorname{Ker}(S)$ is finite dimensional and its image $\operatorname{Im}(S)$ has finite codimension in $F$. We will use the basic fact that $\sigma_{e}(S)=\{\lambda \in \mathbf{C}: \lambda-S \notin \Phi(E)\}$ for $S \in L(E)$. We first state the following simple fact:

Lemma 1. Let $0<\theta<1$. Then

$$
e_{j} \notin \operatorname{Im}\left(r_{\theta}-S_{[\theta]}\right), \quad j \in \mathbf{Z},
$$

so that $r_{\theta}-S_{[\theta]} \notin \Phi\left(\left(X_{0}, X_{1}\right)_{[\theta]}\right)$.

Proof. Let $n \in \mathbf{Z}$ and suppose that $\left(a_{j}\right) \in\left(X_{0}, X_{1}\right)_{[\theta]}$ satisfies $\left(r_{\theta}-S_{[\theta]}\right)\left(a_{j}\right)=\left(r_{\theta} a_{j}-\right.$ $\left.a_{j-1}\right)_{j \in \mathbf{Z}}=e_{n}$. Thus

$$
r_{\theta} a_{m}-a_{m-1}=0 \quad \text { for } m \neq n, \quad r_{\theta} a_{n}-a_{n-1}=1 \text {. }
$$

We get by iteration from (2) that $a_{n+k}=r_{\theta}^{-k} a_{n}$ for $k=1,2, \ldots$ Since $\sum_{k=1}^{\infty} r_{\theta}^{2(n+k)}\left|a_{n+k}\right|^{2}=\left|a_{n}\right|^{2} \sum_{k=1}^{\infty} r_{\theta}^{2 n}$ is finite we must have $0=a_{n}=a_{n+k}$ for all $k=1,2, \ldots$. By substituting $a_{n}=0$ into (2) we get that $a_{n-k}=-r_{\theta}^{k-1}$ for $k=1,2, \ldots$ This yields a contradiction since

$$
\sum_{k=n+1}^{\infty} r_{\theta}^{2(n-k)}\left|a_{n-k}\right|^{2}=\sum_{k=n+1}^{\infty} r_{\theta}^{2(n-k)} r_{\theta}^{2(k-1)}=\infty .
$$

Proposition 1. $\sigma_{e}\left(T_{[\theta]}\right)=\left\{\lambda \in \mathbf{C}:|\lambda|=r_{\theta}\right\}$ for $0<\theta<1$ and $\theta \neq \frac{1}{2}$, and $\sigma_{e}\left(T_{[1 / 2]}\right)=$ $\{\lambda \in \mathbf{C}:|\lambda| \leq 1\}$. Hence the map $\theta \mapsto \sigma_{e}\left(T_{[\theta]}\right)$ is discontinuous at $\theta=\frac{1}{2}$.

Proof. Case $\theta=\frac{1}{2}$. Suppose first that $0<|\lambda| \leq 1$. Let $e_{n} \in\left(X_{0}, X_{1}\right)_{[1 / 2]}=\ell^{2}(\mathbf{Z})$ be the $n$-th (non-normalized) unit coordinate vector for $n \in \mathbf{Z}$. Assume that $n \in \mathbf{Z}$ and $\left(x_{j}\right) \in\left(H_{0}, H_{1}\right)_{[1 / 2]}=\ell^{2}\left(\mathbf{Z},\left(X_{0}, X_{1}\right)_{[1 / 2]}\right)$ satisfies

$$
\begin{aligned}
\left(\lambda-T_{[1 / 2]}\right)\left(x_{j}\right) & =\left(\ldots, \lambda x_{0}-S x_{-1}, \lambda x_{1}-(S-I) x_{0}, \lambda x_{2}-S x_{1}, \ldots\right) \\
& =\left(\ldots, 0, e_{n}, 0,0, \ldots\right),
\end{aligned}
$$

where $e_{n}$ occupies the 1 -st coordinate in $\ell^{2}\left(\mathbf{Z},\left(X_{0}, X_{1}\right)_{[1 / 2]}\right)$. Here we put $S=S_{[1 / 2]}$ for notational simplicity. Hence we get

$$
\lambda x_{j}-S x_{j-1}=0 \quad \text { for } j \neq 1, \quad \lambda x_{1}-(S-I) x_{0}=e_{n} .
$$

By iteration in (3) starting from $x_{2}=\lambda^{-1} S x_{1}$ we get $x_{k+1}=\lambda^{-k} S^{k} x_{1}$ for $k=1,2, \ldots$ Thus $\left\|x_{k+1}\right\|=|\lambda|^{-k}\left\|x_{1}\right\| \geq\left\|x_{1}\right\|$ since the right shift operator $S$ is an isometry on $\ell^{2}(\mathbf{Z})$. This yields that $x_{1}=0$, so that $x_{j}=0$ for $j \geq 1$. Hence we are left with the condition $(S-I) x_{0}=-e_{n}$ in (3). However, this is not possible according to Lemma 1. This means that the image $\operatorname{Im}\left(\lambda-T_{[1 / 2]}\right)\left(\ell^{2}\left(\mathbf{Z},\left(X_{0}, X_{1}\right)_{[1 / 2]}\right)\right.$ has infinite codimension in $\ell^{2}\left(\mathbf{Z},\left(X_{0}, X_{1}\right)_{[1 / 2]}\right)$, and $\lambda \in \sigma_{e}\left(T_{[1 / 2]}\right)$ whenever $0<|\lambda| \leq 1$. The case $\lambda=0$ is obvious, since $(3)$ yields directly that $(S-I) x_{0}=-e_{n}$. 
Case $\theta \neq \frac{1}{2}$. Suppose that $|\lambda|=r_{\theta}$ and let $\left(e_{n}\right)_{n \in \mathbf{Z}}$ be the (non-normalized) coordinate basis in $\left(X_{0}, X_{1}\right)_{[\theta]}$. Let $n \in \mathbf{N}$ and assume that $\left(x_{j}\right) \in\left(H_{0}, H_{1}\right)_{[\theta]}=\ell^{2}\left(\mathbf{Z},\left(X_{0}, X_{1}\right)_{[\theta]}\right)$ satisfies

$$
\begin{aligned}
\left(\lambda-T_{[\theta]}\right)\left(x_{j}\right) & =\left(\ldots, \lambda x_{0}-S_{[\theta]} x_{-1}, \lambda x_{1}-\left(S_{[\theta]}-I\right) x_{0}, \lambda x_{2}-S_{[\theta]} x_{1}, \ldots\right) \\
& =\left(\ldots, 0, e_{n}, 0,0, \ldots\right),
\end{aligned}
$$

with $e_{n}$ in the 1 -st coordinate in $\ell^{2}\left(\mathbf{Z},\left(X_{0}, X_{1}\right)_{[\theta]}\right)$. Hence,

$$
\lambda x_{j}-S_{[\theta]} x_{j-1}=0 \quad \text { for } j \neq 1, \quad \lambda x_{1}-\left(S_{[\theta]}-I\right) x_{0}=e_{n} .
$$

The facts that $x_{k+1}=\lambda^{-k} S_{[\theta]}^{k} x_{1}$ for $k=1,2, \ldots$ by (4), and $\left\|S_{[\theta]} x\right\|_{[\theta]}=r_{\theta}\|x\|_{[\theta]}$ for $x \in\left(X_{0}, X_{1}\right)_{[\theta]}$, yield that $\left\|x_{k+1}\right\|_{[\theta]}=|\lambda|^{-k} r_{\theta}^{k}\left\|x_{1}\right\|_{[\theta]}=\left\|x_{1}\right\|_{[\theta]}$. Hence $x_{1}=0$ and $\left(S_{[\theta]}-I\right) x_{0}=-e_{n}$ for $n \in \mathbf{Z}$ in (4). Thus $\lambda-T_{[\theta]} \notin \Phi\left(\ell^{2}\left(\mathbf{Z},\left(X_{0}, X_{1}\right)_{[\theta]}\right)\right)$ by Lemma 1 for $|\lambda|=r_{\theta}$. This implies the claim, since $\sigma\left(T_{[\theta]}\right) \subset\left\{\lambda \in \mathbf{C}:|\lambda|=r_{\theta}\right\}$ for $\theta \neq \frac{1}{2}$ by $[\mathrm{AM}$, Example 1].

By following the outline of [AM, Thm. 2] one may prove the following stronger discontinuity property for $\sigma_{e}\left(T_{[\theta]}\right)$ (the details are left to the interested reader):

FACT. Let $M \subset(0,1)$ be a dense $G_{\delta}$-set. Then there is a couple $\left(H_{0}, H_{1}\right)$ consisting of non-separable Hilbert spaces and $T \in L\left(H_{0}+H_{1}\right)$ so that $M$ is the set of continuity of the map $\theta \mapsto \sigma_{e}\left(T_{[\theta]}\right)$.

Log-convexity of the essential norm in real interpolation. Cwikel [C] showed, solving a longstanding problem, that $T_{\theta, p}$ is a compact operator $\left(E_{0}, E_{1}\right)_{\theta, p} \rightarrow\left(F_{0}, F_{1}\right)_{\theta, p}$ whenever $T_{0}: E_{0} \rightarrow F_{0}$ or $T_{1}: E_{1} \rightarrow F_{1}$ is compact. Recently, Cobos, Fernandez-Martinez and Martinez [CMM] established a quantitative strengthening of his result. Let

$$
\gamma(S)=\inf \left\{\varepsilon>0: S B_{E} \subset D+\varepsilon B_{F}, D \subset F \text { compact }\right\}
$$

be the measure of non-compactness of $S \in L(E, F)$, where $B_{E}$ is the closed unit ball of $E$. They showed [CMM, Thm. 1.2] that $\gamma\left(T_{\theta, p}\right)$ is logarithmically convex up to a multiplicative constant, that is,

$$
\gamma\left(T_{\theta, p}\right) \leq 16 \delta \cdot \gamma\left(T_{0}\right)^{1-\theta} \gamma\left(T_{1}\right)^{\theta}
$$

for $T \in L\left(E_{0}+E_{1}, F_{0}+F_{1}\right)$, where $\delta=\delta(\theta)=\frac{2^{\theta}}{3-2^{\theta}-2^{1-\theta}}$. The constant $16 \delta$ in (5) cannot be replaced by 1, see [CMM, Example 1.1]. Note also that $\delta(\theta) \rightarrow \infty$ as $\theta \rightarrow 0+$.

Equation (5) raises the problem whether the essential norm is also logarithmically convex in real interpolation. Here $\gamma(S) \leq\|S\|_{e}$ for any $S$, but $\|\cdot\|_{e}$ and $\gamma(\cdot)$ are not equivalent in general, see [AT, Thms. 2.3 and 2.5] and [T2, Thm. 1.2]. We note also that $r_{e}\left(T_{\theta, p}\right) \leq r_{e}\left(T_{0}\right)^{1-\theta} r_{e}\left(T_{1}\right)^{\theta}$ for $0<\theta<1$ by [CMM, Cor. 1.3], where $r_{e}(S)=\max \{|\lambda|$ : $\left.\lambda \in \sigma_{e}(S)\right\}$ is the essential spectral radius of $S \in L(E)$. We refer to e.g. [LS], [AT], $[\mathrm{T} 2]$ and $[\mathrm{A}]$ for further results related to measures of non-compactness and the essential spectral radius.

We show that there is an analogue of (5) for $\left\|T_{\theta, p}\right\|_{e}$ provided that certain compact approximation conditions are satisfied. A Banach space $X$ is said to have the inner compact approximation property (abbreviated inner CAP) if there is a constant $C<\infty$ so that 


$$
\inf \{\|U-U V\|: V \in K(X),\|I-V\| \leq C\}=0
$$

for any compact operator $U \in K(X, Z)$ (where $Z$ is an arbitrary Banach space). Moreover, $X$ has the bounded compact approximation property (BCAP) if there is a constant $C<\infty$ so that for any compact subset $D \subset X$ and $\varepsilon>0$ there is a compact operator $V \in K(X)$ satisfying

$$
\sup _{x \in D}\|x-V x\|<\varepsilon \text { and }\|I-V\| \leq C .
$$

The preceding compact approximation properties differ from the standard approximation properties defined in terms of finite rank operators. For instance, Willis [W] constructed a space $X$ so that $X$ has the BCAP but $X$ fails to have the approximation property AP. There is also a space $Y$ having a Schauder basis which fails to have the inner CAP, see [GW, 4.3], [T2, Example 2.5] or [CJ, Thm. 2.5]. We refer e.g. to [GW], [S], [CJ] or [T2] for further examples of this kind.

Let $E, F$ be Banach spaces. It is convenient to put

$$
\begin{array}{r}
\beta(S)=\inf \{\varepsilon>0:\|S x\| \leq\|U x\|+\varepsilon\|x\| \text { for all } x \in E, \\
U \in K(E, Z), Z \text { an arbitrary Banach space }\},
\end{array}
$$

for $S \in L(E, F)$. It is known [GM] that $\beta(S)=\gamma\left(S^{*}\right)$ for $S \in L(E, F)$. We will need the estimates

$$
\frac{1}{2} \gamma(S) \leq \beta(S)=\gamma\left(S^{*}\right) \leq 2 \gamma(S), \quad S \in L(E, F),
$$

due to Goldenstein and Markus (see e.g. [T2, Prop. 2.3.(i)]).

Theorem 1. Let $p \in[1, \infty]$ be fixed. Assume that $\left(E_{0}, E_{1}\right)$ and $\left(F_{0}, F_{1}\right)$ are interpolation couples, so that either

(i) the interpolation spaces $\left(F_{0}, F_{1}\right)_{\theta, p}$ have the BCAP with a uniformly bounded constant $C$ for $0<\theta<1$, or

(ii) the interpolation spaces $\left(E_{0}, E_{1}\right)_{\theta, p}$ have the inner $C A P$ with a uniformly bounded constant $C$ for $0<\theta<1$.

Then,

$$
\left\|T_{\theta, p}\right\|_{e} \leq 32 C \delta \cdot\left\|T_{0}\right\|_{e}^{1-\theta}\left\|T_{1}\right\|_{e}^{\theta}
$$

for $T \in L\left(E_{0}+E_{1}, F_{0}+F_{1}\right)$ and $0<\theta<1$, where $\delta=\delta(\theta)>0$ is the constant from (5). Proof. (i) If $\left(F_{0}, F_{1}\right)_{\theta, p}$ has the BCAP with constant $C$, then $\|S\|_{e} \leq C \cdot \gamma(S)$ for any $S \in$ $L\left(Z,\left(F_{0}, F_{1}\right)_{\theta, p}\right)$ and any $Z$, see $\left[\mathrm{LS}\right.$, Thm. 3.6]. By applying this to $T_{\theta, p}:\left(E_{0}, E_{1}\right)_{\theta, p} \rightarrow$ $\left(F_{0}, F_{1}\right)_{\theta, p}$ we get from $(5)$ that

$$
\left\|T_{\theta, p}\right\|_{e} \leq C \cdot \gamma\left(T_{\theta, p}\right) \leq 16 C \delta \cdot \gamma\left(T_{0}\right)^{1-\theta} \gamma\left(T_{1}\right)^{\theta} \leq 16 C \delta \cdot\left\|T_{0}\right\|_{e}^{1-\theta}\left\|T_{1}\right\|_{e}^{\theta}
$$

for $T \in L\left(E_{0}+E_{1}, F_{0}+F_{1}\right)$.

(ii) If $\left(E_{0}, E_{1}\right)_{\theta, p}$ has the inner CAP with constant $C$, then $\|S\|_{e} \leq C \cdot \beta(S)$ for any $S \in L\left(\left(E_{0}, E_{1}\right)_{\theta, p}, Z\right)$ and any $Z$ by [T2, Thm. 1.2]. Hence, by applying (5) to $T_{\theta, p}$ : $\left(E_{0}, E_{1}\right)_{\theta, p} \rightarrow\left(F_{0}, F_{1}\right)_{\theta, p}$ and using (6) we get

$$
\begin{aligned}
\left\|T_{\theta, p}\right\|_{e} & \leq C \cdot \beta\left(T_{\theta, p}\right) \leq 2 C \cdot \gamma\left(T_{\theta, p}\right) \leq 32 C \delta \cdot \gamma\left(T_{0}\right)^{1-\theta} \gamma\left(T_{1}\right)^{\theta} \\
& \leq 32 C \delta \cdot\left\|T_{0}\right\|_{e}^{1-\theta}\left\|T_{1}\right\|_{e}^{\theta} .
\end{aligned}
$$


Theorem 1 is much simpler to state in special cases where one of the couples is trivial (that is, $E_{0}=E_{1}$ or $F_{0}=F_{1}$ ). Here (5) was known earlier (see [TE, Thm. 1]) with a uniformly bounded constant $c=c(\theta)$.

Corollary 1. Let $p \in[1, \infty]$ be fixed, let $E$ be a Banach space and $\left(F_{0}, F_{1}\right)$ a Banach couple. Suppose that either

(i) $\left(F_{0}, F_{1}\right)_{\theta, p}$ have the BCAP with a uniformly bounded constant $C$ for $0<\theta<1$, or

(ii) $E$ has the inner $C A P$ with constant $C$.

Then (7) holds with a uniformly bounded constant for $T \in L\left(E, F_{0}+F_{1}\right)$.

Corollary 2. Let $p \in[1, \infty]$ be fixed, let $F$ be a Banach space and $\left(E_{0}, E_{1}\right)$ a Banach couple. Suppose that either

(i) $\left(E_{0}, E_{1}\right)_{\theta, p}$ have the inner $C A P$ with a uniformly bounded constant $C$ for $0<\theta<1$, or

(ii) F has the BCAP with constant $C$.

Then (7) holds with a uniformly bounded constant for $T \in L\left(E_{0}+E_{1}, F\right)$.

The preceding approximation assumptions on the interpolation spaces have some drawbacks.

REMARK 1. Neither the BCAP nor the inner CAP passes in general to real interpolation spaces, so that the conditions on $\left(E_{0}, E_{1}\right)_{\theta, p}$ or $\left(F_{0}, F_{1}\right)_{\theta, p}$ in Theorem 1 cannot be ensured by assuming that the spaces $E_{0}, E_{1}$ or $F_{0}, F_{1}$ have these properties. Indeed, it follows from [DS, Thm. 1] (see also [GMS, p. 505]) that there exists a Banach couple $\left(E_{0}, E_{1}\right)$ so that $E_{0}$ and $E_{1}$ have the BCAP, but $\left(E_{0}, E_{1}\right)_{\theta, p}$ fail to have the BCAP for any $0<\theta<1$ and $1 \leq p \leq \infty$. Similarly, there is $\left(E_{0}, E_{1}\right)$ so that $E_{0}$ and $E_{1}$ have the inner CAP, but $\left(E_{0}, E_{1}\right)_{\theta, p}$ fails to have the inner CAP for any $0<\theta<1$ and $1 \leq p \leq \infty$.

REMARK 2. Another convexity estimate for $\left\|T_{\theta, p}\right\|_{e}$ can be found by modifying an argument of Teixeira and Edmunds [TE, Thm. 2]. Here one assumes that the image couple $\left(F_{0}, F_{1}\right)$ satisfies a technical approximation condition $(\mathrm{H})$ (we refer to [TE, p. 133] for the definition).

FACT. Let $0<\theta<1$ and $1 \leq p \leq \infty$. Assume that $\left(E_{0}, E_{1}\right)$ and $\left(F_{0}, F_{1}\right)$ are Banach couples, where $\left(F_{0}, F_{1}\right)$ satisfies condition $(H)$ with the constants $c_{0}$ and $c_{1}$. Then

$$
\left\|T_{\theta, p}\right\|_{e} \leq c_{0}^{1-\theta} c_{1}^{\theta}\left\|T_{0}\right\|_{e}^{1-\theta}\left\|T_{1}\right\|_{e}^{\theta}
$$

for $T \in L\left(E_{0}+E_{1}, F_{0}+F_{1}\right)$.

We omit the details, since condition $(\mathrm{H})$ appears to be quite cumbersome to verify and its connection to Theorem 1 remains unclear. Note that at least $\left(L^{p_{0}}(0,1), L^{p_{1}}(0,1)\right)$ and $\left(\ell^{p_{0}}, \ell^{p_{1}}\right)$ satisfy condition $(\mathrm{H})$ for $1 \leq p_{0}, p_{1}<\infty$, see [TE, p. 135].

REMARK 3. The inequality $\left\|S_{\theta, p}\right\|_{e} \leq c \cdot\left\|S_{0}\right\|_{e}^{1-\theta}\left\|S_{1}\right\|_{e}^{\theta}$ cannot always hold with $c=1$. This fact is a simple modification of [CMM, Example 1].

Let $1<q<\infty, \theta=1-\frac{1}{q}$ and consider the couples $\left(\ell^{1}, \ell^{1}\right),\left(\ell^{1}, \ell^{\infty}\right)$ and the identity operator $S=I$. We need the fact that $\left\|I: \ell^{1} \rightarrow \ell^{\infty}\right\|_{e} \leq \frac{1}{2}$. Indeed, define the rank-1 operator $U: \ell^{1} \rightarrow \ell^{\infty}$ by $U x=\frac{1}{2}\left(\sum_{j=1}^{\infty} x_{j}\right)(1,1, \ldots)$ for $x=\left(x_{j}\right) \in \ell^{1}$, and note that 
$\|I x-U x\|_{\infty}=\sup _{n \in \mathbf{N}}\left|x_{n}-\frac{1}{2} \sum_{j \neq n}^{\infty} x_{j}\right| \leq \frac{1}{2}$ for $x=\left(x_{k}\right) \in B_{\ell^{1}}$. Since Corollary 1 applies to these couples, the estimates in [CMM, pp. 27-28] yield then that

$$
1=\left\|I: \ell^{1} \rightarrow \ell^{q}\right\|_{e} \leq c \cdot \theta^{-(1-\theta)}\left\|I: \ell^{1} \rightarrow \ell^{\infty}\right\|_{e}^{\theta} \leq c \cdot \theta^{-(1-\theta)} 2^{-\theta},
$$

where $c<\infty$. By letting $q \rightarrow \infty$ in (9) we see that $c>1$.

Theorem 1 raises the problem whether the compact approximation conditions are essential for the logarithmic convexity of $\left\|T_{\theta, p}\right\|_{e}$. The proof of (5) in [CMM, Thm. 1.2] uses (among other things) the facts that

$$
\gamma(S Q)=\gamma(S) \quad \text { and } \quad \frac{1}{2} \gamma(S) \leq \gamma(J S) \leq \gamma(S), \quad S \in L(E, F),
$$

where $Q: X \rightarrow E$ is a linear metric surjection (that is, $\overline{Q B_{X}}=B_{E}$ ) and $J: F \rightarrow Y$ is an isometric embedding. In addition, a couple of crucial estimates from the argument in [CMM, Thm. 1.2] would also work in the case of $\|\cdot\|_{e}$ provided that the seminorms $\gamma(\cdot)$ and $\|\cdot\|_{e}$ are uniformly comparable between suitable sequence spaces.

The equivalence of $\gamma(\cdot)$ and $\|\cdot\|_{e}$ is closely tied to the BCAP or the inner CAP, see [AT, Thm. 2.5] and [T2, Thm. 1.2] . The same remark also applies to the analogues of (10) for $\|\cdot\|_{e}$ (see the next section). The preceding facts suggest the following intriguing possibility, which in part motivated this note.

Question. Find Banach couples $\left(E_{0}, E_{1}\right),\left(F_{0}, F_{1}\right)$ and a sequence of operators $\left(T_{n}\right) \subset$ $L\left(E_{0}+E_{1}, F_{0}+F_{1}\right)$ so that

$$
\lim _{n \rightarrow \infty} \frac{\left\|\left(T_{n}\right)_{0}\right\|_{e}^{1-\theta} \cdot\left\|\left(T_{n}\right)_{1}\right\|_{e}^{\theta}}{\left\|\left(T_{n}\right)_{\theta, p}\right\|_{e}}=0
$$

holds for any $0<\theta<1$ (or, for some $0<\theta<1$ ). Here $p \in[1, \infty]$ is fixed.

Behaviour of $\|\cdot\|_{e}$ under isometric embeddings and metric surjections. Results in $[\mathrm{AT}]$ and [T2] imply that the essential norm may behave quite strangely under isometric embeddings and metric surjections. The preceding compact approximation properties are crucial tools for this, but these facts are poorly documented in the literature. Some nonexplicit examples are contained in [AT, Thm. 3.5 and Cor. 3.6] (see also [T3, Example 1.5]). The unpublished thesis [T1, pp. 4-5] contains simpler versions of Examples 2 and 3 below, but other variants are also possible. Hence we take the opportunity to include these results here. Put $S_{E}=\{x \in E:\|x\|=1\}$.

EXAMPLE 2. Let $F$ be a separable Banach space that fails to have the BCAP. Fix a countable subset $\Lambda=\left\{x_{n}^{*}: n \in \mathbf{N}\right\} \subset S_{F^{*}}$ so that $\Lambda$ norms $F$, and let $J: F \rightarrow \ell^{\infty}$ be the isometry $J x=\left(x_{n}^{*}(x)\right), x \in F$. We claim that there is a space $E$ and a sequence $\left(S_{n}\right) \subset L(E, F)$ for which

$$
\left\|S_{n}\right\|_{e}=1 \quad \text { for } n \in \mathbf{N} \quad \text { and } \quad\left\|J S_{n}\right\|_{e} \rightarrow 0 \quad \text { as } n \rightarrow \infty .
$$

Indeed, [AT, Thms. 2.3 and 2.5] provide a Banach space $E$ and a sequence $\left(S_{n}\right) \subset L(E, F)$ for which $\left\|S_{n}\right\|_{e}=1$ and $\gamma\left(S_{n}\right)<\frac{1}{n}$ for $n \in \mathbf{N}$. Let $J_{\infty}: F \rightarrow \ell^{\infty}\left(B_{F^{*}}\right)$ be the isometry $J_{\infty} x=\left(x^{*}(x)\right)_{x^{*} \in B_{F^{*}}}$ for $x \in F$. It is known that $\left\|J_{\infty} S\right\|_{e}=\gamma\left(S^{*}\right)$ for $S \in L(E, F)$ by 
[As, Cor. 5.6]. Hence it follows from (6) that

$$
\left\|J_{\infty} S_{n}\right\|_{e}=\gamma\left(S_{n}^{*}\right) \leq 2 \cdot \gamma\left(S_{n}\right)<\frac{2}{n}, \quad n \in \mathbf{N} .
$$

We factorize $J_{\infty}=J_{0} \circ J$, where $J_{0}$ is a suitable linear isometry $\ell^{\infty} \rightarrow \ell^{\infty}\left(B_{F^{*}}\right)$, by viewing $\ell^{\infty}=\ell^{\infty}(\Lambda) \subset \ell^{\infty}\left(B_{F^{*}}\right)$ as a closed one-complemented subspace. Recall that there is a norm one projection $P: \ell^{\infty}\left(B_{F^{*}}\right) \rightarrow J_{0}\left(\ell^{\infty}\right)$ by the extension property of $\ell^{\infty}$. Thus $\left\|J S_{n}\right\|_{e}=\left\|P J_{\infty} S_{n}\right\|_{e}<\frac{2}{n} \rightarrow 0$ as $n \rightarrow \infty$, which yields (11).

EXAmple 3. Let $E$ be a separable Banach space that fails to have the inner CAP. Fix a countable dense subset $\left\{x_{n}: n \in \mathbf{N}\right\}$ of $S_{E}$, and let $Q: \ell^{1} \rightarrow E$ be the quotient map defined by $Q\left(\sum_{n=1}^{\infty} a_{n} e_{n}\right)=\sum_{n=1}^{\infty} a_{n} x_{n}$ for $\sum_{n=1}^{\infty} a_{n} e_{n} \in \ell^{1}$. By [T2, Thm. 1.2] there is a space $F$ and a sequence $\left(S_{n}\right) \subset L(E, F)$ so that $\left\|S_{n}\right\|_{e}=1$ and $\beta\left(S_{n}\right)<\frac{1}{n}$ for $n \in \mathbf{N}$. The fact that $Q$ is a quotient map implies that $\|S Q\|_{e}=\gamma(S)$ for $S \in L(E, F)$, see $[$ As, Thm. 3.8 and Cor. 3.9]. Hence it follows from (6) that $\left\|S_{n} Q\right\|_{e}=\gamma\left(S_{n}\right) \leq 2 \cdot \beta\left(S_{n}\right) \rightarrow 0$ as $n \rightarrow \infty$.

Conversely, compact approximation properties guarantee that $\|S Q\|_{e}\left(\right.$ or $\left.\|J S\|_{e}\right)$ is uniformly comparable to $\|S\|_{e}$.

Proposition 2. (i) Let E, $F$ and $Z$ be Banach spaces, and $J: F \rightarrow Z$ a fixed linear isometric embedding. Then, there is $c>0$ so that

$$
c\|S\|_{e} \leq\|J S\|_{e}, \quad S \in L(E, F),
$$

provided one of the following conditions are satisfied:

(a) E has the inner $C A P$, (b) $F$ has the BCAP, or (c) there is a bounded linear projection $P: Z \rightarrow J E$.

(ii) Let $E, F$ and $Z$ be Banach spaces, and $Q: Z \rightarrow E$ a fixed linear metric surjection. Then, there is $c>0$ so that

$$
c\|S\|_{e} \leq\|S Q\|_{e}, \quad S \in L(E, F),
$$

provided one of the following conditions are satisfied:

(a) E has the inner CAP, (b) F has the BCAP, or (c) there is a bounded linear projection $P: Z \rightarrow \operatorname{Ker}(Q)$.

Proof. (i) Suppose that either $F$ has the BCAP or that $E$ has the inner CAP. It follows from [LS, Thm. 3.6], respectively [T2, Thm. 1.2] and (6), that there is $c>0$ so that $\|S\|_{e} \leq c \cdot \gamma(S) \leq 2 c \cdot \gamma(J S) \leq 2 c \cdot\|J S\|_{e}$ for $S \in L(E, F)$. Let $P$ be a bounded projection $Z \rightarrow J E$ and assume that $\lambda>\|J S\|_{e}$. Pick $R \in K(E, Z)$ so that $\|J S-R\|<\lambda$. Then $J^{-1} P R$ defines a compact operator $E \rightarrow F$ satisfying

$$
\left\|S-J^{-1} P R\right\|=\|J S-P R\|=\|P(J S-R)\|<\|P\| \lambda .
$$

Thus $\|S\|_{e} \leq\|P\| \cdot\|J S\|_{e}$ for $S \in L(E, F)$.

(ii) The argument for the cases (a) and (b) is similar to that of the first part of (i), since $\gamma(S Q)=\gamma(S)$ for $S \in L(E, F)$. 
Put $M=\operatorname{Im}(I-P)$, where $P: Z \rightarrow \operatorname{Ker}(Q)$ is a projection. Suppose that $\lambda>\|S Q\|_{e}$ and pick $R \in K(Z, F)$ satisfying $\|S Q-R\|<\lambda$. Note that $\widetilde{Q}=Q_{\mid M}: M \rightarrow Q M=E$ has a bounded inverse $\widetilde{Q}^{-1}: E \rightarrow M$. If $J: M \rightarrow Z$ denotes the inclusion map, then

$$
\begin{aligned}
\left\|S-R J \widetilde{Q}^{-1}\right\| & =\left\|S Q J \widetilde{Q}^{-1}-R J \widetilde{Q}^{-1}\right\| \\
& \leq\|S Q-R\| \cdot\left\|\widetilde{Q}^{-1}\right\|<\lambda \cdot\left\|\widetilde{Q}^{-1}\right\|,
\end{aligned}
$$

where $R J \widetilde{Q}^{-1} \in K(E, F)$.

\section{References}

[A] A. G. Aksoy, The radius of the essential spectrum, J. Math. Anal. Appl. 128 (1987), 101-107.

[AM] E. Albrecht and V. Müller, Spectrum of interpolated operators, Proc. Amer. Math. Soc. 129 (2001), 807-814.

[As] K. Astala, On measures of noncompactness and ideal variations in Banach spaces, Ann. Acad. Sci. Fenn. Ser. AI Math. Dissertationes 29 (1980), 1-42.

[AT] K. Astala and H.-O. Tylli, On the bounded compact approximation property and measures of non-compactness, J. Funct. Anal. 70 (1987), 388-401.

[BL] J. Bergh and J. Löfström, Interpolation Spaces, Springer-Verlag, 1976.

[BS] C. Bennett and R. C. Sharpley, Interpolation of Operators, Academic Press, 1988.

[CJ] P. G. Casazza and H. Jarchow, Self-induced compactness in Banach spaces, Proc. Roy. Soc. Edinburgh Sect. A 126 (1996), 355-362.

[CMM] F. Cobos, P. Fernández-Martinez and A. Martinez, Interpolation of the measure of non-compactness by the real method, Studia Math. 135 (1999), 25-38.

[C] M. Cwikel, Real and complex interpolation and extrapolation of linear operators, Duke Math. J. 65 (1992), 333-343.

[DS] S. J. Dilworth and D. Sobecki, On hereditariness for real and complex interpolation, Far East J. Math. Sci. 5 (1997), 91-98.

[GMS] D. J. H. Garling and S. J. Montgomery-Smith, Complemented subspaces of spaces obtained by interpolation, J. London Math. Soc. 44 (1991), 503-513.

[GM] M. González and A. Martinon, Operational quantities derived from the norm and measures of non-compactness, Proc. R. Irish Acad. 91A (1991), 63-70.

[GW] N. Grønbæk and G. A. Willis, Approximate identities in Banach algebras of compact operators, Canad. Math. Bull. 36 (1993), 45-53.

[KM] N. Kalton and S. Montgomery-Smith, Interpolation of Banach spaces, in: Handbook of the Geometry of Banach Spaces, vol. 2, W. B. Johnson and J. Lindenstrauss (eds.), Elsevier, 2003, 1131-1175.

[LS] A. Lebow and M. Schechter, Semigroups of operators and measures of noncompactness, J. Funct. Anal. 7 (1971), 1-26.

[S] C. Samuel, Bounded approximate identities in the algebra of compact operators on a Banach space, Proc. Amer. Math. Soc. 117 (1993), 1093-1096.

[TE] M. F. Teixeira and D. E. Edmunds, Interpolation theory and measures of non-compactness, Math. Nachr. 104 (1981), 129-135.

[T1] H.-O. Tylli, On semiFredholm operators, Calkin algebras and related quantities, Dissertation, University of Helsinki, 1986. 
[T2] H.-O. Tylli, The essential norm of an operator is not self-dual, Israel J. Math. 91 (1995), 93-110.

[T3] H.-O. Tylli, Duality of the weak essential norm, Proc. Amer. Math. Soc. 129 (2001), 1437-1443.

[W] G. A. Willis, The compact approximation property does not imply the approximation property, Studia Math. 103 (1992), 99-108. 